\title{
Bacillus mojavensis RRC101 Lipopeptides Provoke Physiological and Metabolic Changes During Antagonism Against Fusarium verticillioides
}

\author{
A. A. Blacutt, ${ }^{1}$ T. R. Mitchell, ${ }^{2}$ C. W. Bacon, ${ }^{2}$ and S. E. Gold ${ }^{2}$ \\ ${ }^{1}$ University of Georgia, Athens, GA, U.S.A.; and ${ }^{2}$ USDA-ARS TMRU, Russell Research Center, Athens, GA, U.S.A. \\ Accepted 10 August 2016.
}

\begin{abstract}
The mycotoxigenic pathogen Fusarium verticillioides threatens the quality and utility of maize across industrial and agricultural purposes. Chemical control is complicated by the intimate endophytic lifestyle of the pathogen with its host. Bacillus mojavensis RRC101, a maize-endophytic bacterium, has been observed to reduce $F$. verticillioides disease severity and fumonisin accumulation when coinoculated to maize. Genome sequencing and annotation identified a number of biocontrol-relevant pathways in RRC101. Biochemical assays confirmed the presence and activity of surfactin- and fengycintype lipopeptides, with fengycins responsible for antifungal activity against $F$. verticillioides. This antagonism manifests as inhibition of filamentous growth, with microscopy revealing hyphal distortions, vacuolization, and lysis. F. verticillioides secondary metabolism also responds to antagonism, with lipopeptide challenge inducing greater fumonisin production and, in the case of fengycins, eliciting pigment accumulation at sites of inhibition. Together, these data suggest that antibiotic and toxin production are components of a complex biochemical interaction among maize endophytes, one pathogenic and one beneficial.
\end{abstract}

Fusarium verticillioides is a seed- and soil-borne fungal pathogen of maize endemic to regions where its host is cultivated (Munkvold et al. 1997). F. verticillioides undergoes a period of asymptomatic endophytic growth, reportedly capable of benefitting the host until induced to grow pathogenically-a process theorized to be triggered by environmental stresses such as drought (Lee et al. 2009). Through infection and subsequent production of the fumonisin mycotoxins, $F$. verticillioides threatens all uses of corn by decreased ethanol yields, toxicity to livestock, and links to human cancers and birth defects (Bothast et al. 1992; Marasas et al. 1981; United States Food and Drug Administration 2001; Voss et al. 2002). Importantly, limits on acceptable fumonisin contamination for maize imports by other countries threaten maize exports from the United States (Miller et al. 1995).

The endemic nature of $F$. verticillioides, its close association with corn, and its adaptation as a soil microbe render chemical controls impractical or prohibitively expensive, with

Corresponding author: S. E. Gold; E-mail: scott.gold@ars.usda.gov

*The $\boldsymbol{e}$-Xtra logo stands for "electronic extra" and indicates that two supplementary figures and two supplementary videos are published online.

This article is in the public domain and not copyrightable. It may be freely reprinted with customary crediting of the source. The American Phytopathological Society, 2016. little demonstrated control difference between organic and conventional farming practices (Ariño et al. 2007). Thus, the value of potential biological control agents to control $F$. verticillioides is readily apparent, provided that such biorationals are host-benign, persistent, and efficacious in their control. With growing consumer awareness of sustainable, low-input agricultural methods, the need for well-understood, effective biological control agents is clear (Batte et al. 2007).

Members of the genus Bacillus are heavily represented among biocontrol agents (BCA), owing to the ubiquity of the genus and its members' resilience and genetic tractability; these are typically strains of $B$. thuringiensis, B. amyloliquefaciens, and B. subtilis (Cawoy et al. 2011). Several Bacillus BCA have been observed to produce lipopeptide-type antibiotics, which are comprised of a peptide ring attached to a hydrocarbon tail of varying length and saturation (Ongena et al. 2005; Ramos et al. 2006) (Fig. 1). Lipopeptides are amphiphilic molecules, exhibiting varying surfactant potency in addition to antibiotic activity (Ongena and Jacques 2008). Generally, these antibiotics are stable in a $\mathrm{pH}$ range from 2 to 10 and retain activity after being heated to $100^{\circ} \mathrm{C}$ for $30 \mathrm{~min}$, making them an extremely durable agent of biocontrol (Zhang et al. 2012).

Bacillus mojavensis is a species closely related to B. subtilis, with most isolates originating from soil and rhizosphere samples (Roberts et al. 1994; Xu and Côté 2003). B. mojavensis RRC101 was isolated from a surface-sterilized maize kernel and has since been observed to internally colonize maize roots and aerial tissues (Bacon and Hinton 2002). This endophytism is significant when taken with RRC101's reported ability to mitigate mycotoxin accumulation and disease inflicted by $F$. verticillioides, with antifungal activity observable in vitro (Bacon and Hinton 2011a; $\mathrm{Hu}$ et al. 2009). RRC101 has also been observed to promote growth in Arabidopsis, presumed to be the result of metabolites secreted by the bacterium (M. Rath personal communication).

Collectively, B. mojavensis strains have been identified as producing members of each of the three predominant classes of lipopeptides; RRC101 was previously described as producing surfactins, with initial reports describing them as essential to biocontrol in the maize- $F$. verticillioides pathosystem (Bacon and Hinton 2011b; Ma et al. 2012). In addition to diverse lipopeptide production, $B$. mojavensis strains have been found to colonize coffee and cacao phyllospheres, suggesting another Bacillus species potentially rich in biocontrol agents intimately associated with their hosts, although multihost colonization data have yet to be published (Jacobsen 2010; Melnick et al. 2008; Nair et al. 2002). Endophytic colonization of host plants could enable targeted, direct delivery of biocontrol activity and crop protection, a useful advance for low-input agriculture and integrated control practices (Batte et al. 2007). 
The work described here assesses biocontrol-relevant secondary metabolism genes present in the $B$. mojavensis RRC101 genome and identifies fengycins as the principal class of secondary metabolites responsible for in vitro antifungal activity against $F$. verticillioides. Under assay conditions, fengycin antagonism is primarily characterized by growth inhibition. Biochemical and microscopic analyses have identified that symptoms of antagonism in $F$. verticillioides involve hyphal swelling and explosive lysis, along with significant alterations to secondary metabolism. Together, these data collectively describe the RRC101$F$. verticillioides biocontrol interaction as a molecular "conversation" among maize endophytes, with $F$. verticillioides' secondary metabolism responding to lipopeptide antagonism.

\section{RESULTS}

The B. mojavensis RRC101 genome possesses

biocontrol-relevant secondary metabolism pathways.

RAST annotation and subsequent bidirectional BLAST to National Center for Biotechnology Information GenBank identified a number of biosynthetic clusters for endophytism- and biocontrol-relevant secondary metabolites (Table 1). Genomic regions with $>85 \%$ identity to known surfactin and fengycin biosynthetic clusters were presumed to be lipopeptide synthetase

\section{Surfactin (C-16)}

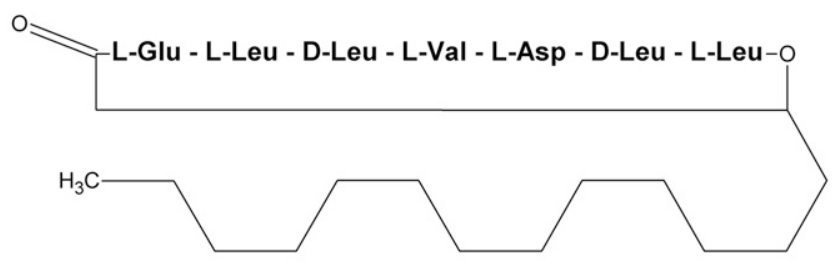

Fengycin A (C-16)

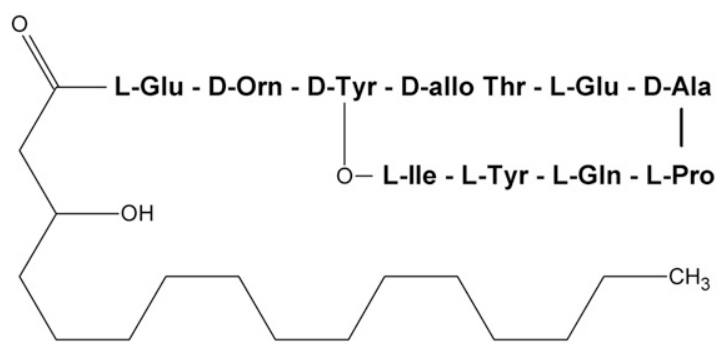

Fig. 1. Chemical diagrams of the lipopeptides surfactin and fengycin A produced by Bacillus mojavensis RRC101. Amino acids comprising the peptide ring are denoted by their three-letter designations. operons. Other biocontrol-relevant secondary metabolite operons identified through sequence analysis include a catechol-type bacillibactin-like siderophore, the antibiotic bacilysin, and a yetunidentified polyketide synthase cluster resembling that of bacillaene (Weber et al. 2015). In addition to secondary metabolites, the RRC101 genome possessed a number of genes encoding products likely involved in host colonization; these include several putative cell wall-degrading enzymes and an expansin protein with high identity to one promoting root colonization in $B$. subtilis (Kerff et al. 2008). As B. mojavensis RRC101 is currently genetically intractable, biochemical means were employed to assay activity of these secondary metabolite clusters.

\section{B. mojavensis produces diverse fengycin isoforms responsible for in vitro antagonism of $F$. verticillioides.}

Primary C-18 fractionation of RRC101 culture supernatants yielded several antagonistic fractions, which were confirmed by highpressure liquid chromatography-mass spectrometry (HPLC-MS) to be enriched for fengycin and surfactin lipopeptides (Fig. 2). To assess the antifungal contribution of individual lipopeptide classes, active primary fractions were pooled for secondary fractionation to produce isolated RRC101 fengycins and surfactins. Utilizing the fragmentation patterns and nomenclature assigned by Pathak et al. (2012), collision-induced dissociation fragmentation spectra of fengycins suggest that $B$. mojavensis $\mathrm{RRC} 101$ produces several variants of fengycins A, B, and C (Table 2, Supplementary Fig. S1). Fengycin-containing preparations exhibited the same in vitro antibiosis as crude extracts, with purified surfactins lacking visible antagonism and failing to inhibit $F$. verticillioides growth (Fig. 3, inset). Antagonistic activity of secondary fractions closely followed total fengycin content (Fig. 4).

\section{Fengycin-mediated antagonism}

of $\boldsymbol{F}$. verticillioides triggers aberrant hyphal morphology.

Microscopy revealed that the leading edges of fengycinchallenged cultures exhibited hyphal aberrations, even at subinhibitory concentrations. The primary microscopic phenotype of fengycin exposure is altered hyphal morphology, characterized by a sinuous growth habit as well as presence of enlarged cell compartments or "swellings" (Fig. 5). Swellings ranged from engorgement of conidia (average $8.60 \pm 2.0 \mu \mathrm{m}$, untreated average $3.99 \pm 0.67 \mu \mathrm{m}, 100$ measurements each) and hyphae to fully swollen intercalary or terminal "bulbs" (average dimensions $23.22 \pm 5.59 \times 30.69 \pm 0.70 \mu \mathrm{m}$ ) (Fig. 5). Swollen hyphal compartments were frequently observed to erupt by violent lysis; conidia were not observed to exhibit this behavior (Fig. 6, Supplementary Videos S1 and S2). Symptoms of antagonism were primarily observed on colony surfaces proximal to antagonistic fraction spots, suggesting that such swellings are a direct consequence of fengycin exposure via diffusion. These observed effects are consistent with the current proposed mechanism

Table 1. Biocontrol-relevant genes in Bacillus mojavensis RRC101

\begin{tabular}{|c|c|c|c|}
\hline Metabolite/enzyme & Role in rhizosphere competence/biocontrol & Reference & PEG Number ${ }^{y}$ \\
\hline Surfactin & Biofilm and host colonization, host defense induction & Ongena and Jacques 2008 & 205 to 208 \\
\hline Fengycin & Antifungal activity & Ongena and Jacques 2008 & 1,874 to 1,881 \\
\hline Subtilosin & Bacteriocin antibiotic & & 3,746 to 3,753 \\
\hline Bacilysin & Antibiotic & & 3,789 to 3,785 \\
\hline Bacillibactin & Siderophore-iron sequestration/mineralization & Dertz et al. 2006 & 3,158 to 3,162 \\
\hline Quercetin dioxygenase ${ }^{\mathrm{z}}$ & Protection from host flavonoids, rhizoplane colonization & Hirooka and Fujita 2010 & $4,042,4,043$ \\
\hline Acetoin reductase & Putative host growth promotion/defense induction & Ryu et al. 2004 & $474,1,917$ \\
\hline Phytase $^{\mathrm{z}}$ & Nutrient availability, growth promotion & Idriss et al. 2002 & 2,048 \\
\hline Expansin $^{\mathrm{z}}$ & Host wall manipulation, facilitates root colonization & Kerff et al. 2008 & 1,914 \\
\hline Pectate lyase ${ }^{z}$ & Facilitates host colonization, potential defense manipulation & Kovtunovych et al. 1999 & $627,1,916$ \\
\hline
\end{tabular}

y Genome 1329377.3 on PubSEED.

${ }^{\mathrm{z}}$ Putatively secreted as designated by Phobius. 
of membrane permeabilization induced by lipopeptide activity (Deleu et al. 2008).

\section{Fengycin-induced hyphal inflation involves} cell-wall expansion and extensive vacuolization ending in cell rupture.

Observation of fengycin-induced bulbs in F. verticillioides M3125::GFP under fluorescence microscopy detected strong green fluorescent protein (GFP) signal within enlarged hyphae, suggesting that bulb formation involved inflation of the entire hyphal compartment. Fluorescent signal retention within cellwall boundaries suggested that fengycin-induced swelling was not immediately lethal to the cell and that the cell wall and plasma membrane had maintained integrity (Fig. 7C). Bulb formation was consistently accompanied by the presence of one or more enlarged organelles, putatively identified as vacuoles by CMAC (7-amino-4-chloromethylcoumarin) staining (Fig. 7F).

While bulbs initially exhibited significant GFP signal and are, thus, presumed metabolically active, lysis was frequently observed and was confirmed using calcofluor and FM4-64 stains. Calcofluor staining of cell walls highlighted postrupture deformation of $F$. verticillioides compartments (Fig. 7B). Presumptively lysed hyphae frequently exhibited an intense red signal throughout the compartment, suggestive of an influx of FM4-64 owing to compromised membranes (Fig. 7D) (Druzhinina et al. 2011). These lysed structures exhibited little-to-no GFP fluorescence; as synthetic GFP has a reported half-life of $18 \mathrm{~h}$, this suggests cytoplasmic leakage and diffusion rather than degradation (Fig. 7C) (Ruijter et al. 2003).

\section{Lipopeptide challenges differentially} alter $\boldsymbol{F}$. verticillioides secondary metabolism.

Second to growth inhibition, the most obvious symptom of fengycin-based antagonism is a band of brown-to-purple pigmentation at the antagonized colony edge (Supplementary Fig. S2). Given that wild-type coloration of $F$. verticillioides M3125 on potato dextrose agar (PDA) is orange-yellow, the color change is attributable to accumulation of either fusarubins (previously referred to as purple perithecial pigment in $F$. fujikuroi) or bikaverin (Medentsev et al. 2005; Studt et al. 2012).

Lipopeptide-challenged $F$. verticillioides cultures accumulated fumonisins at higher levels than controls, in a manner correlated with lipopeptide concentration (Table 3). Despite the lack of antifungal activity, the "high" rate ( $20 \times$ concentration) of surfactins provoked a statistically significant increase in total fumonisin content, whereas the lower rate $(10 \times$ concentration) of surfactin had no significant effect. To assess whether lipopeptide antagonism disrupted fumonisin export, plugs were compared with those taken directly outside of the colony growth; no statistically significant differences were observed between control and treatment groups for the ratio of colony internal/external fumonisins (not shown). These data suggest that the increased fumonisin accumulation is a genuine increase in production and not an artifact of impaired export. In addition to increased overall fumonisin content, the fengycin-challenged cultures exhibited slightly altered fumonisin isoform composition. The ratio of $\mathrm{FB}_{1}$ to total fumonisins (sum of $\mathrm{FB}_{1}, \mathrm{FB}_{2}$, and $\mathrm{FB}_{3}$ ) decreased as fengycin concentration increased. While

Table 2. Observed fengycin diversity in Bacillus mojavensis RRC101

\begin{tabular}{lclc}
\hline Fengycin & Major ions & Peptide sequence $^{z}$ & Lipid chain lengths $^{\text {Marn }}$ \\
\hline A & 1080,966 & EOrnYTEAPQYI & 14 to 17 \\
A2 & 1066,952 & EOrnYTEAPQYV & 16,17 \\
B & 1108,994 & EOrnYTEVPQYI & 15 to 19 \\
B2 & 1094,980 & EOrnYTEVPQYV & 16,17 \\
C & 1008,1122 & EOrnYTEIPQYI & 16 to 18 \\
\hline
\end{tabular}

${ }^{\mathrm{z}}$ Bolded amino acid positions indicate differences between isoforms.

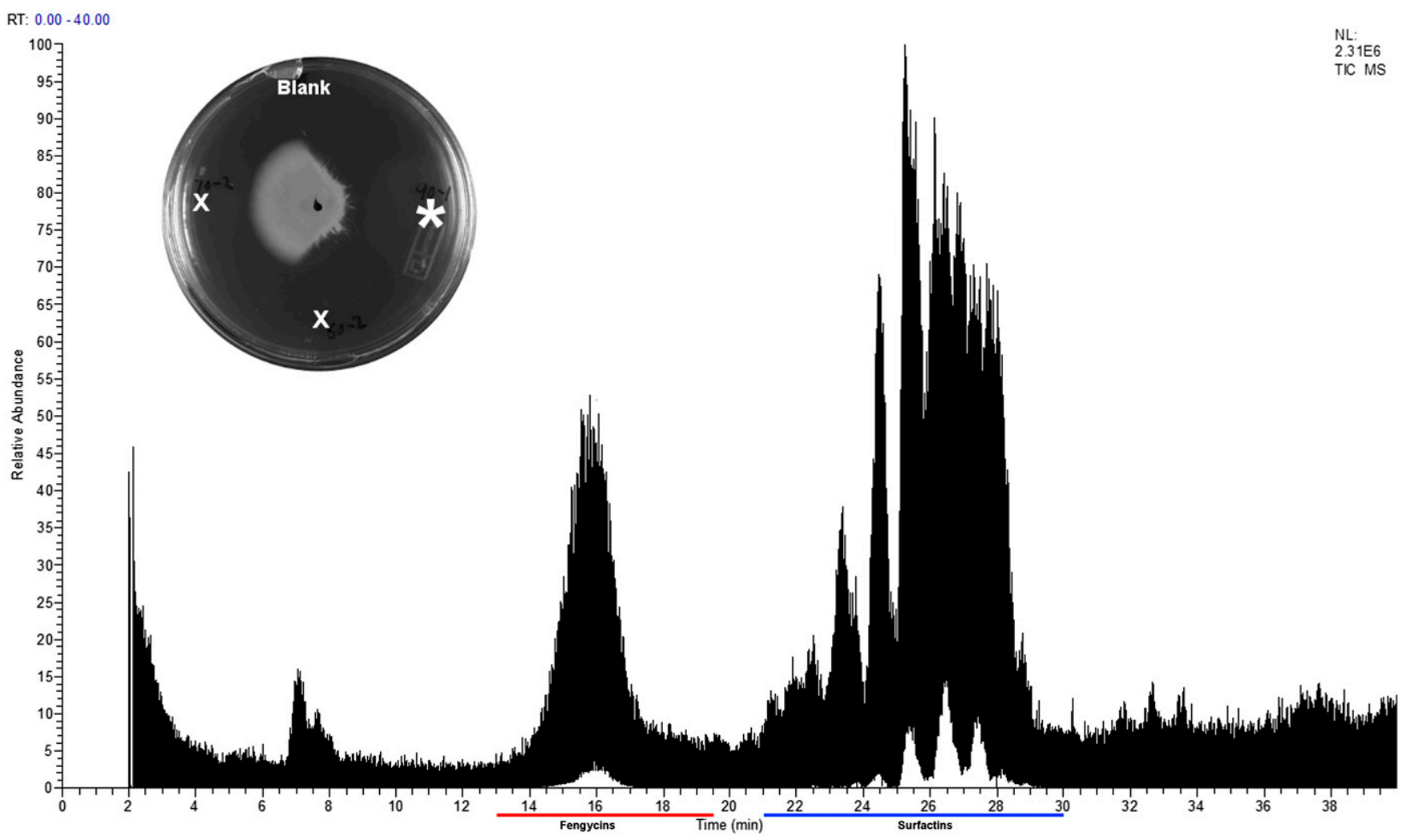

Fig. 2. Total ion chromatogram of an antagonistic fraction (demonstrated in inset plate photo) as identified through high-pressure liquid chromatography-mass spectrometry. Regions corresponding to fengycins and surfactins are underlined and labeled. Inset: Antagonism assay plate demonstrating antifungal activity of the depicted fraction (asterisk) as compared with methanol (Blank) and two inactive fractions (X), the effect of lipopeptide exposure on radial growth. 
the high-surfactin treatment induced fumonisin accumulation equivalent to that of the low-fengycin ( $10 \times$ concentration) treatment, the distributions of fumonisin isoforms were statistically indistinguishable from controls.
LaeA is a transcription factor identified in Aspergillus spp. as controlling secondary metabolism; in Fusarium, mutants for the orthologous $L A E 1$ have previously been described as diminished in fumonisin and bikaverin expression (Bok and

A

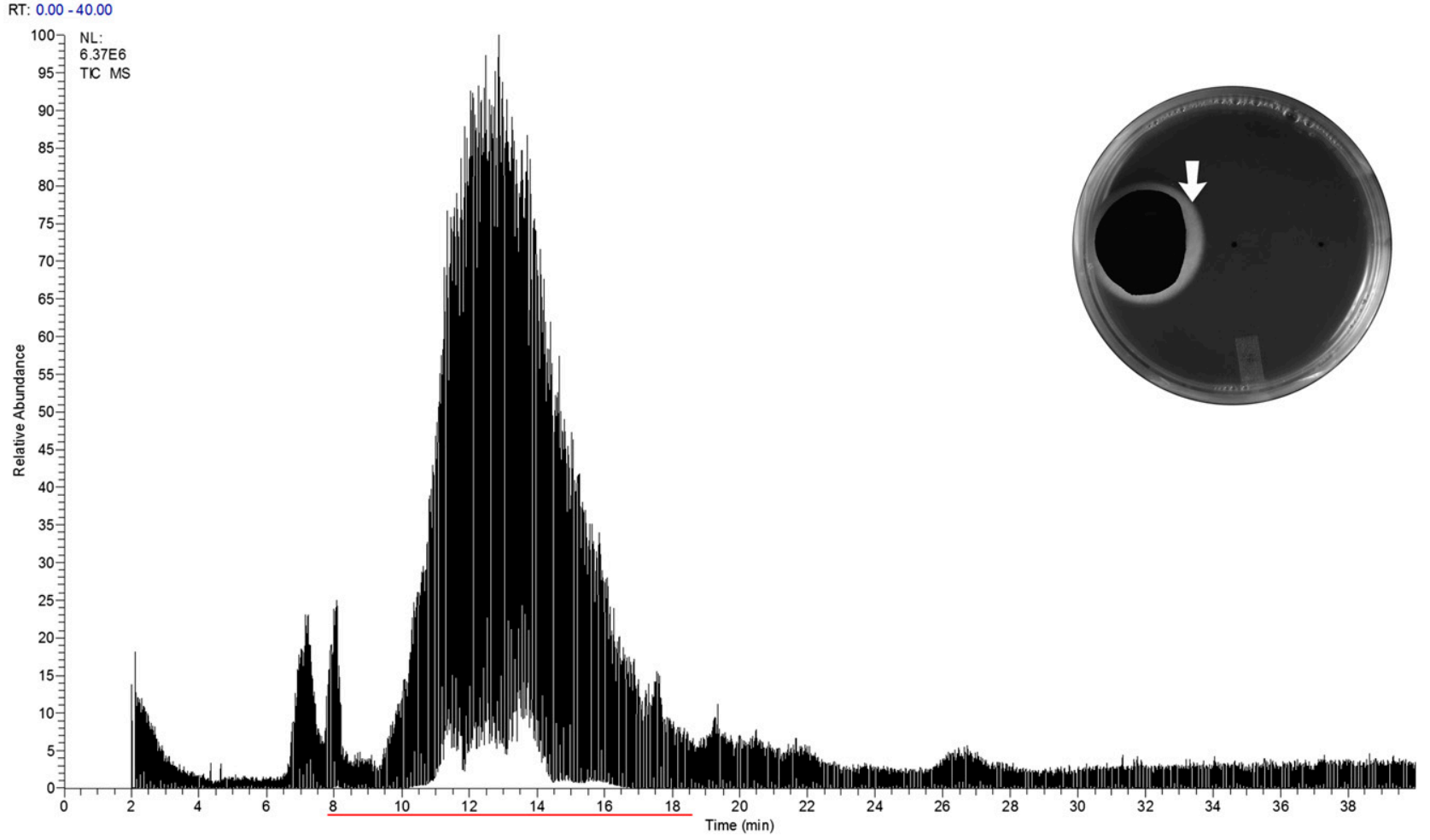

B

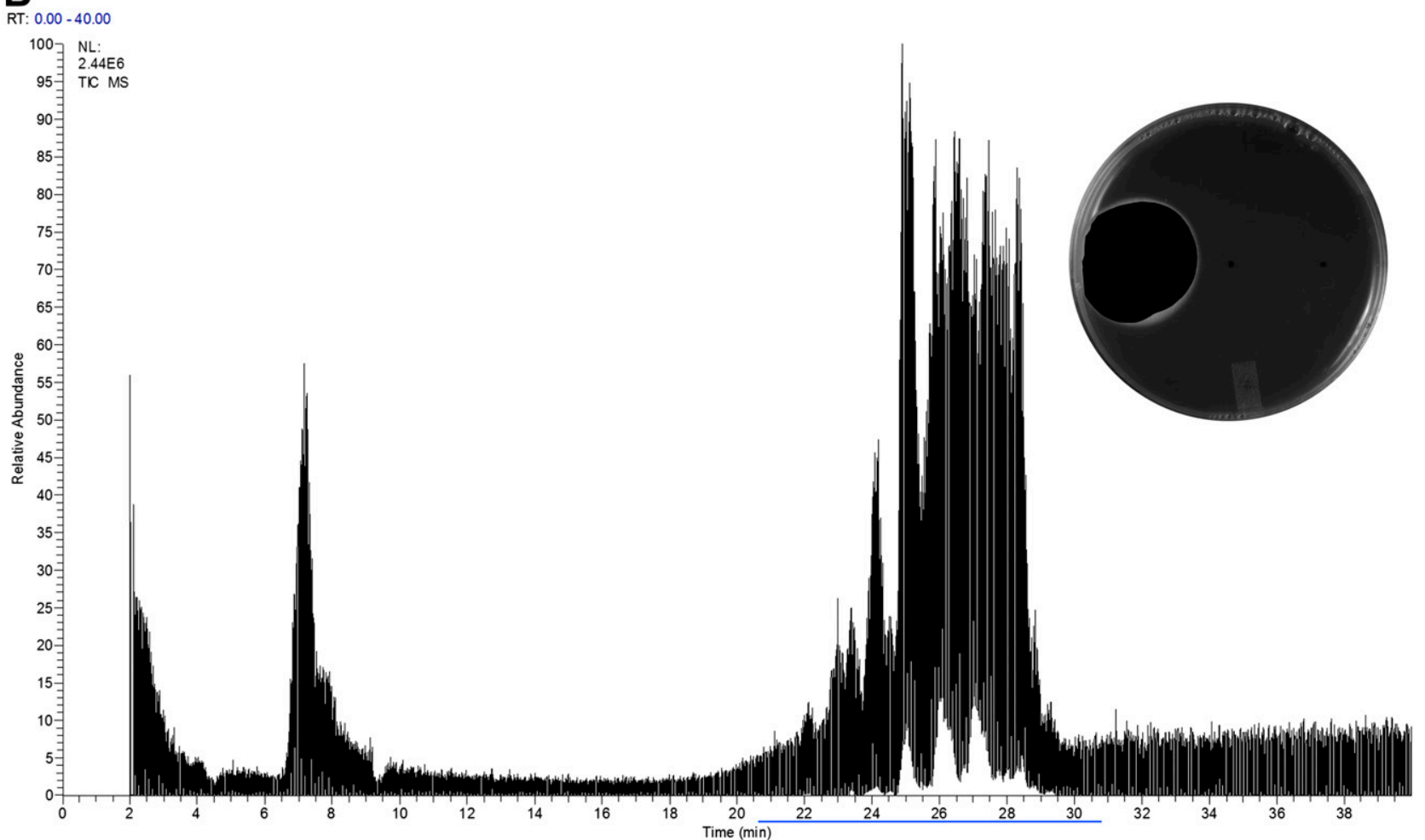

Fig. 3. High-pressure liquid chromatography-mass spectrometry spectra of collected RRC101 A, fengycin and B, surfactin fractions, with individual overlapping peaks representing the varied isoforms in each sample. Representative antagonism plates are inset to each of the spectra, with silhouettes of the respective fractions (in black) superimposed on that of the methanol control (white). The purified surfactins exhibited no significant antagonism, whereas the fengycins produced clear antagonism (indicated by the white arrow). 
Keller 2004; Butchko et al. 2011). Antagonism assays conducted with a $\Delta$ Lael strain of $F$. verticillioides detected fumonisins at concentrations below limit of quantitation across all treatments and controls (not shown).

\section{DISCUSSION}

The genus Bacillus contains a number of plant-associated biocontrol agents; $B$. mojavensis is among relatively few described species observed to exhibit endophytic growth traits, and strain RRC101, in particular, colonizes maize aerial tissues. Genomic data from confirmed endophytes may reveal common trends and elucidate the underpinnings of such associations with plant hosts, such as secretory system losses or the presence of hydrolytic enzymes (Reinhold-Hurek and Hurek 2011). Sequencing data revealed that the RRC101 genome is replete with clues to the mechanisms of such associations (Gold et al. 2014). Several annotated genes in RRC101 are predicted to function similar to genes essential to host colonization in phytopathogenic and endophytic bacteria alike, notably pectate lyase and expansin (Table 1). Pectate lyase produced by phytopathogens facilitates host colonization through the primary activity of cell-wall digestion; byproducts from such activity are known to modulate host defense pathways (Collmer and Keen 1986; Kovtunovych et al. 1999; Wegener et al. 1996). Cell wall-degrading enzymes and bacterial expansins (observed to enhance pliability of plant cell walls) produced by phytopathogens and B. subtilis, respectively, have been demonstrated to act on host cell walls to facilitate colonization; in the case of B. subtilis, expansin deletion impaired colonization of maize roots (Georgelis et al. 2014; Kerff et al. 2008). RRC101 possesses genes for synthesis of the hormones indole acetic acid and 2,3-butanediol; these growth-promoting phytohormones have been observed in both beneficial and phytopathogenic bacteria (Spaepen and Vanderleyden 2011). Annotation and study of gene repertoires in plant-associated microbes, particularly beneficial endophytes like RRC101, can aid in identifying functions involved in plant colonization strategies common to phyllosphere inhabitants. Knowledge of these colonization strategies will facilitate marker development for screening putative biocontrol agents and may prove useful in designing control strategies against current and future pathogens. Along with putative endophytism factors in the RRC101 genome are operons coding for both surfactin and fengycin synthetases, of which the latter are responsible for the in vitro antibiosis observed against F. verticillioides.
Bacterial lipopeptide classes have conditionally efficacious antagonism potential, suggesting that activity of lipopeptidebased biocontrol is broadly dependent on both lipopeptide and target membrane compositions. Surfactins have demonstrated hemolytic properties as well as activity against enveloped viruses, some bacteria, and mosquito larvae; fengycins and iturins are potently antifungal, with varying activities against other organisms (Ongena and Jacques 2008; Geetha and Manonmani 2010). Target membrane sterol composition and lipid organization influence these interactions, as do the amino acid composition and lipid tail characteristics of the lipopeptides (Bonmatin et al. 2003). If activity is indeed determined by specific membrane and lipopeptide interactions, these variations could present selective pressure on the underlying synthetases and lead to variable antagonism repertoires at the species or even strain level.

Antagonistic potency of RRC101 fengycin extracts against F. verticillioides does not perfectly follow the total fengycin content of fractions (Fig. 4). The reverse-phase fractioning, paired with this result, suggest that later eluting (more hydrophobic) fengycin isoforms possess greater antifungal activity against $F$. verticillioides in plate assays (Fig. 3, inset). This may be due to differences in peptide or lipid composition, or both, resulting in improved agar diffusion or stronger isoform-specific activity against the fungal membrane. Lipopeptide membranespecific activity has been observed among surfactins with regards to perception by plant hosts and subsequent defense induction (Jourdan et al. 2009). Given that both systems rely upon lipopeptide interactions with membranes, it is likely that an analogous preferential interaction exists for antifungal activity. Variation in lipopeptide composition is a result of nonribosomal peptide synthetases' substrate promiscuity, which is, in turn, influenced by sequence diversity at the operon level (Daren Mueller 2014; Shu et al. 2002). If such specialization is occurring within ecological niches, in-depth genetic and biochemical descriptions of the diversity of lipopeptide synthetases and their products become valuable tools in development of efficacious biocontrol strategies.

An experimentally relevant consequence of lipopeptide variability is added difficulty of separation and characterization of individual isoforms. Without tandem mass spectrometry, for example, a parent ion of $1,492.6 \mathrm{~m} / \mathrm{z}$ could represent $\mathrm{C}-18$ fengycin A or C-16 fengycin B. Though reverse phase HPLC fractioning was capable of separating the two classes of lipopeptides from one another, isoform-level separation would require higher-resolution techniques. Such capability is necessary to better evaluate the utility of specific lipopeptide

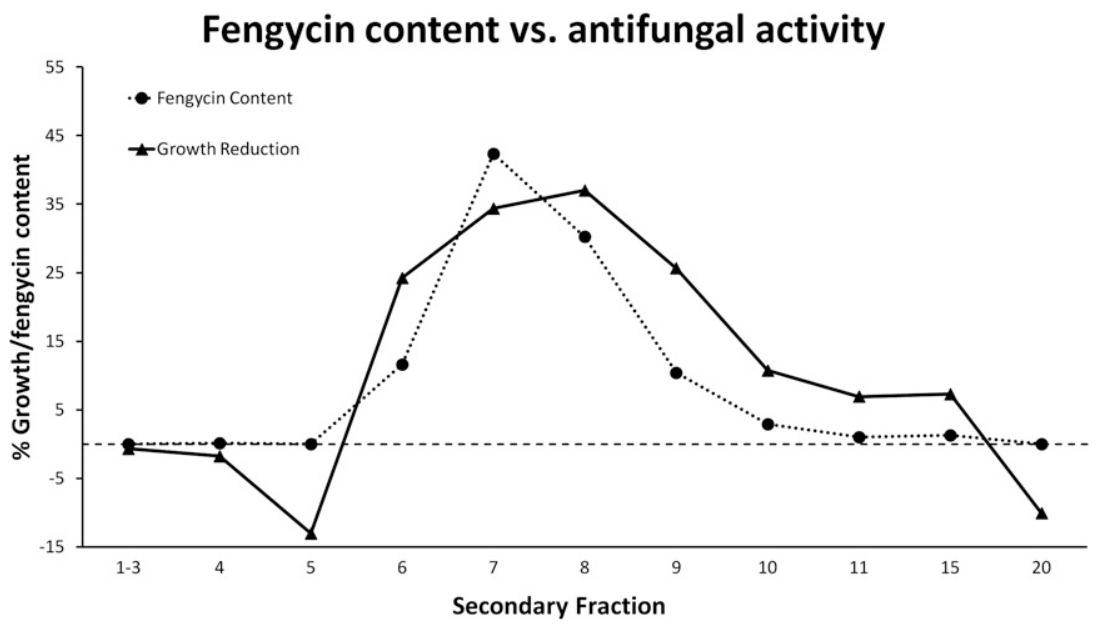

Fig. 4. Correlation of antagonism with fengycin lipopeptides in RRC101 extract fractions. Secondary fractions were compared with the parent antagonistic fraction for growth reduction (triangles, solid line) and liquid chromatography mass spectrometry-derived fengycin content as compared with the sum of all fractions (circles, dotted line). Dashed line $=0$. 
isoforms, which would, in turn, enable more nuanced description of their structure-function relationships.

Existing publications refer to surfactin-class lipopeptides as possessing antifungal activity (Bacon and Hinton 2002; MagetDana et al. 1992). However, most available data describes synergistic activity when surfactin is applied in conjunction with antifungal agents, without substantial evidence for standalone efficacy. By contrast, the antifungal activity of fengycins and iturins produced by $B$. subtilis has been shown to compose most, if not all, of the antifungal activity of the species' lipopeptide arsenal, as appears to be the case in RRC101 (Ongena et al. 2005). Although isolated RRC101 surfactins lacked detectable antifungal activity against $F$. verticillioides in vitro,

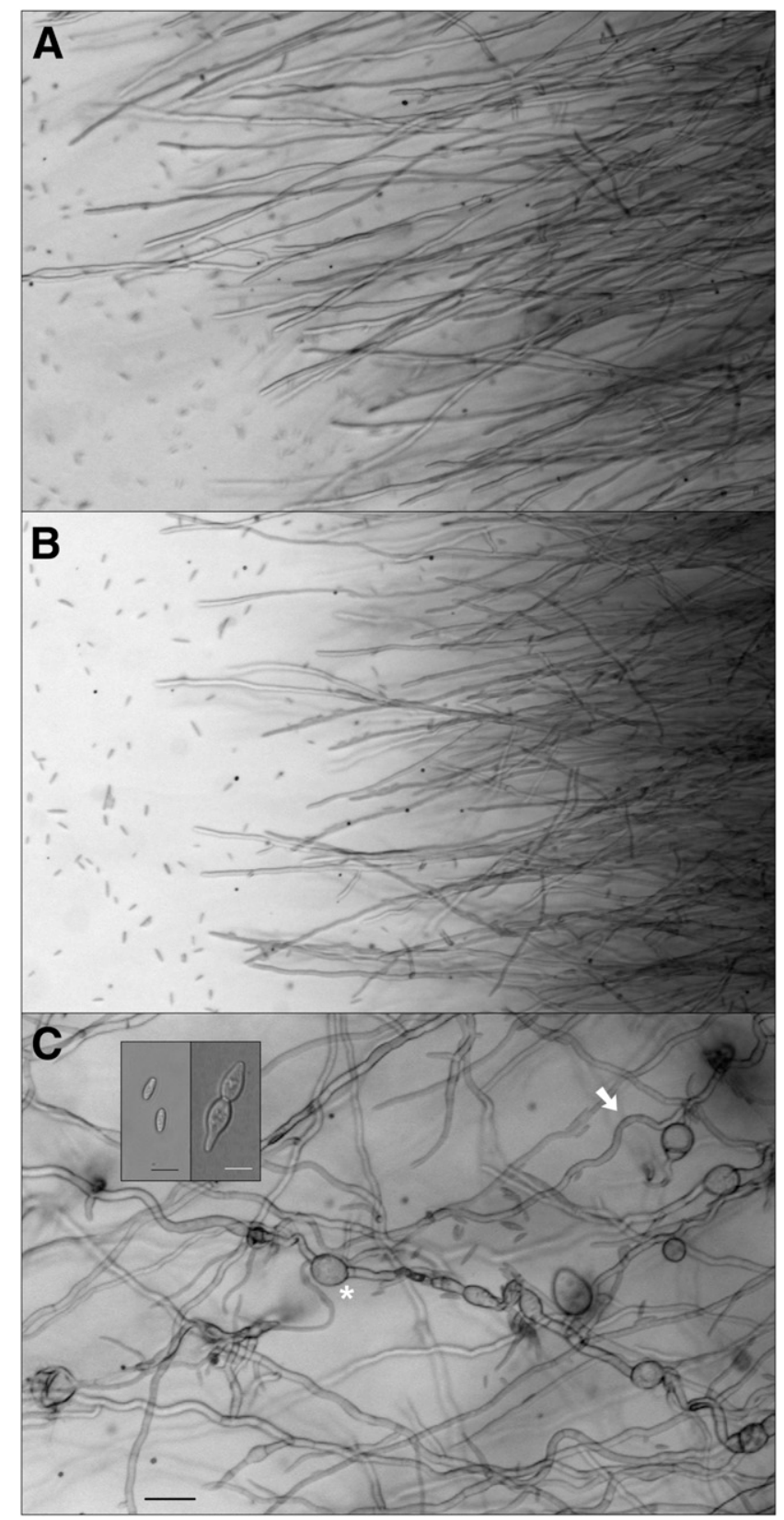

Fig. 5. Morphological response of Fusarium verticillioides to RRC101 lipopeptide challenge. Colony edges of $F$. verticillioides challenged by $\mathbf{A}$, a negative control showing normal hyphal growth and conidiation, B, surfactin extracts indistinguishable from controls, and $\mathbf{C}$, fengycin extracts showing sinuous hyphal growth (arrow) and bulbs (asterisk). Bar $=50 \mu \mathrm{m}$. Inset: Wild-type conidia (left) and conidia exhibiting fengycin-induced swelling (right). Bar $=10 \mu \mathrm{m}$. evidence in other systems suggests that they may act indirectly in biological control. Surfactin production has been tied to root colonization and biofilm formation by Bacillus spp. and may serve to induce host defenses against pathogens (Bais et al. 2004; Henry et al. 2011).

Hyphal bulbs resulting from fengycin exposure consistently contain at least one putative vacuole, as determined through CMAC staining, often representing the majority of the cell volume. Bulb formation and lysis are consistent with the current model of lipopeptide-based antagonism being a result of membrane destabilization through the formation of mixed micelles and pores. Structures induced by lipopeptides have been referred to as chlamydospores in $F$. solani $\mathrm{f}$. sp. radicicola and F. graminearum ( $\mathrm{Li}$ et al. 2012). In contrast, lack of chlamydospore production by $F$. verticillioides is a diagnostic trait of the species (Leslie et al. 2006) and, while fengycin-induced swellings bear a strong resemblance to chlamydospores, their irregular shape, heavy vacuolization, and tendency to lyse suggest against this possibility. This divergence in $F$. verticillioides' lipopeptide response extends to secondary metabolism responses to both surfactins and fengycins.

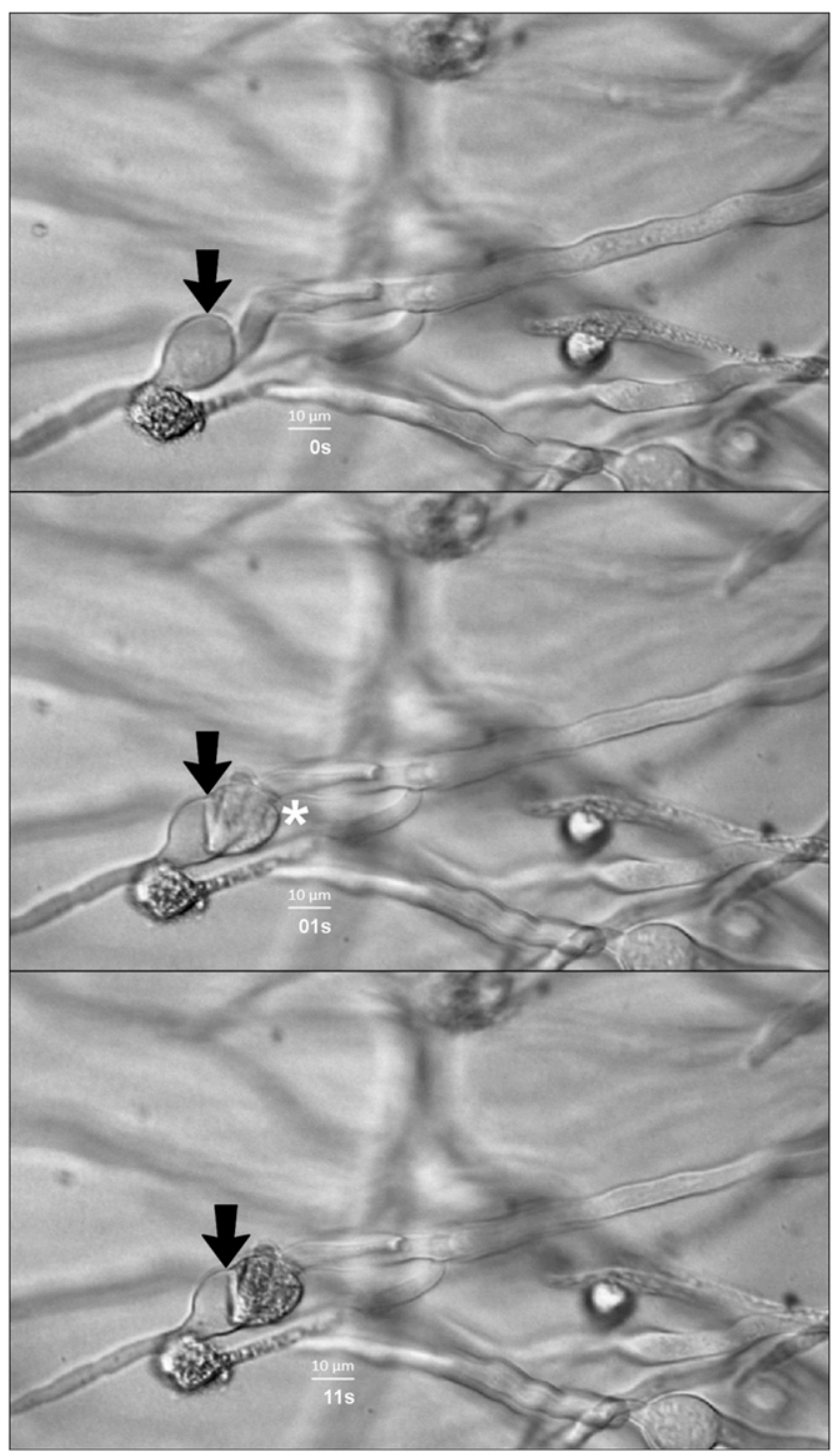

Fig. 6. An example of explosive lysis observed in Fusarium verticillioides cultures challenged with RRC101 fengycins, suggestive of cell-wall dissolution and membrane failure. Arrow = hyphal bulb induced by fengycin challenge, asterisk $=$ explosive lysis accompanied by hyphal ejecta. 
Fusarium verticillioides fumonisin production responds differentially when exposed to each class of lipopeptides. The response to both classes tested was an increased production of fumonisins, with surfactins provoking a milder response. Fengycins uniquely affect not only concentration but fumonisin composition; the increased accumulation of total fumonisins under fengycin challenge is accompanied by a proportional reduction of $\mathrm{FB}_{1}$. The biosynthetic pathway of fumonisins offers a possible explanation; $\mathrm{FB}_{3}$ serves as a precursor in $\mathrm{FB}_{1}$ production, therefore, disproportionate accumulation of $\mathrm{FB}_{3}$ may be a discrepancy in the activities of FUM1 and the converting enzyme FUM3 (Butchko et al. 2006). This may be explained via enzyme kinetics, where the activity of FUM3 is being outpaced by upstream activity in the fumonisin pathway; however, surfactin-based induction can yield similar concentrations without significant perturbation of isoform composition (Table 3).

Differential induction of secondary metabolite pathways suggests that lipopeptide perception and response in $F$. verticillioides is a nuanced physiological process. In experiments performed by Hu et al. (2009), a reduction in fumonisin under fengycin challenge was observed, contrary to the relationship observed here. Differences in assay conditions may explain the discrepancy. The work presented here employed lipopeptide challenges as extracts spotted and allowed to diffuse through agar media, whereas $\mathrm{Hu}$ et al. (2009) directly applied lipopeptides to a growing liquid
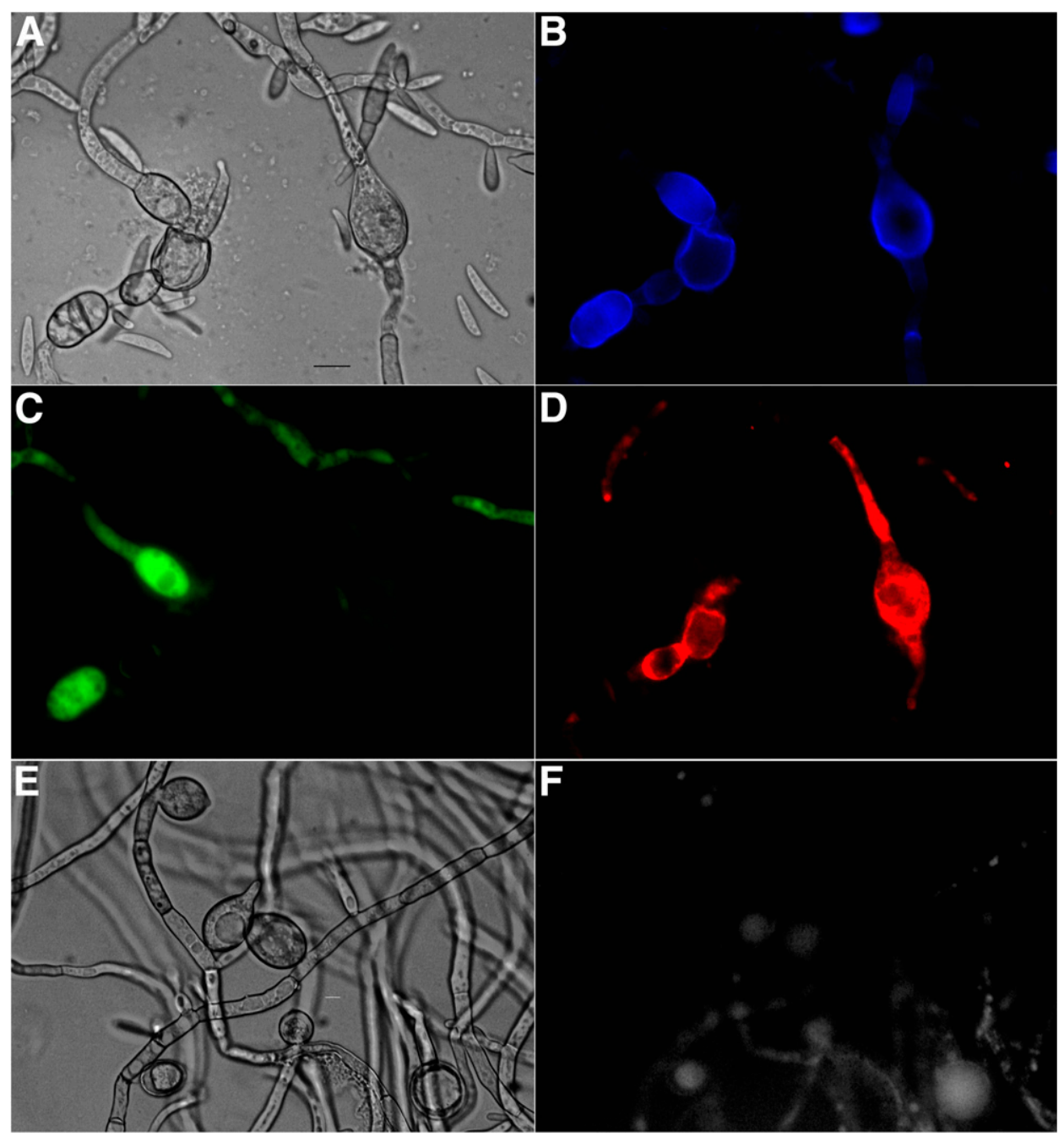

Fig. 7. Fluorescence microscopy of Fusarium verticillioides bulbs produced under challenge from RRC101 fengycins. A, Bright field image of hyphae; B, Calcofluor-labeled cell walls; C, cytosolic green fluorescent protein expression; D, intense FM4-64 staining indicative of destabilized and hyperpermeable membranes as a result of the fengycin treatment. $\mathbf{E}$ and $\mathbf{F}, F$. verticillioides hyphae under fengycin challenge exhibit vacuole-like organelle accumulation under differential interference contrast and fluorescent visualization of the vacuole-labeling dye 7-amino-4-chloromethylcoumarin, respectively. Bar $=20 \mu \mathrm{m}$.

Table 3. Fumonisin production by M 3125 in response to lipopeptide challenge ${ }^{y}$

\begin{tabular}{lcccc}
\hline Treatment & Experiment 1 & Experiment 2 & Experiment 3 & Average FB $_{\mathbf{1}} \mathbf{z}$ \\
\hline Control & $19.6(2.9) \mathrm{c}$ & $18.5(2.1) \mathrm{d}$ & $17.1(1.5) \mathrm{b}$ & $60.41 \mathrm{a}$ \\
$10 \times$ Surfactins & $20.3(1.4) \mathrm{bc}$ & $21.5(1.3) \mathrm{dc}$ & $13.9(4.0) \mathrm{b}$ & $60.04 \mathrm{a}$ \\
20× Surfactins & $28.7(1.2) \mathrm{ab}$ & $32.0(4.1) \mathrm{c}$ & $16.6(2.9) \mathrm{b}$ & $5.88 \mathrm{a}$ \\
$10 \times$ Fengycins & $30.5(8.7) \mathrm{ab}$ & $28.3(2.8) \mathrm{bc}$ & $25.1(4.4) \mathrm{a}$ & $58.56 \mathrm{~b}$ \\
$20 \times$ Fengycins & $34.1(4.2) \mathrm{a}$ & $37.5(3.1) \mathrm{a}$ & $25.9(2.0) \mathrm{a}$ & $57.73 \mathrm{~b}$ \\
\hline
\end{tabular}

${ }^{\mathrm{y}}$ Values are in ppm; letters indicate groups significantly different via Tukey's highly significant difference at $P<0.05$. Standard deviations are in parentheses.

${ }^{\mathrm{z}}$ Average $\mathrm{FB}_{1} \%=$ average ratio of $\mathrm{FB}_{1}$ to total fumonisins. 
culture, followed by incubation. In the experiments in which lipopeptide exposure occurs via the gradual process of diffusion, the results suggest that, at lower concentrations, antagonistic lipopeptides induce $F$. verticillioides to mount a response through secondary metabolism, a recognized phenomenon among subinhibitory concentrations of antibiotics (Davies et al. 2006). Class-specific responses to lipopeptides suggest divergent mechanisms of perception and response in $F$. verticillioides when compared with observations in other fungi.

Both lipopeptide classes produced by RRC101 induced increased fumonisin levels in $F$. verticillioides, with growth inhibition, pigment accumulation, and hyphal distortion observed only following fengycin exposure. $F$. verticillioides colonies antagonized by fengycins accumulate brown-to-purple pigmentation at the leading edge. Pigment production is associated with stress responses in Fusarium species; synthesis of bikaverin (a naphthoquinone possessing limited antimicrobial activity) is induced by nitrogen limitation and acidic $\mathrm{pH}$ (Deshmukh et al. 2014; Limón et al. 2010). Genes for bikaverin synthesis in $F$. verticillioides are also coregulated with heat- and oxidativestress response genes through adenylyl cyclase (Choi and $\mathrm{Xu}$ 2010; Medentsev et al. 2005). The species description for $F$. verticillioides states that chlamydospores are not produced, but enlarged hyphae reminiscent of pseudochlamydospores have been observed (Leslie et al. 2006). However, fengycin-induced bulbs resemble inflated bodies observed in $F$. graminearum when induced to produce tricothecenes (Menke et al. 2013). These $F$. graminearum "toxisomes" are themselves morphologically and functionally analogous to structures originally detailed in Aspergillus parasiticus during aflatoxin production (Chanda et al. 2009). In fengycin-challenged $F$. verticillioides, presence of these structures correlates with increased fumonisin production, but their lysis and general growth inhibition are incongruent with being a specialized structure, as is their absence during surfactininduced fumonisin production.

Hyphal vacuolization and death, both symptoms of fengycin antagonism, also resemble the process of autophagy as described by Pollack et al. (2009): a central autophagosome-containing vacuole appears and may fill an entire hyphal compartment undergoing cell death (Pollack et al. 2009; Richards et al. 2010). Similarly, senescence in Podospora anserina is signaled by increased pigmentation, reduced aerial hyphae, and abnormal hyphal morphology (Sharon et al. 2009). As F. verticillioides undergoes such processes under fengycin challenge, induced senescence may be part of the response to fengycin antagonism. In Verticillium dahliae, cell death pathways were found to be upregulated under iturin antagonism, hinting at a role for such processes in biological control activity (Han et al. 2015). The surfactin-type lipopeptide WH1fungin from B. amyloliquefaciens WH1 has also been observed to induce apoptosis in yeast and Rhizoctonia spp. (Qi et al. 2010). Induced apoptosis as a mechanism of antagonism has also been proposed to exist in the case of Candida albicans, in which the fungus-produced isoprenoid quorum-sensing molecule farnesol induces cell death in Fusarium and Aspergillus species (Semighini et al. 2008).

Differential responses to lipopeptide challenge in $F$. verticillioides suggest the process of antagonism is more complex than direct lysis following plasma membrane pore formation. Such nuance is supported by contrasting the fengycin- and surfactin-shared response of fumonisin accumulation with the fengycin-specific morphological effects and putative bikaverin production. Both bikaverin and fumonisin biosynthetic genes are regulated by Lael, with fumonisin gene expression dropping in deletants (Butchko et al. 2011). Surfactin challenge induces a more modest accumulation of fumonisin than fengycin, without any other effects of fengycin exposure, suggesting the action of a different perception mechanism rather than overt antagonism (Table 3 ).
The fengycin-associated upregulation of secondary metabolism observed via fumonisin and pigment accumulation suggest that the response to fengycin antagonism may be at least partially Lael-dependent. Under bioassay conditions, extracts from the tested $\triangle L A E 1$ strain produced no detectable fumonisin across all treatments but retained pigment accumulation in response to fengycin (not shown). Assays performed by Butchko et al. (2012) comparing wild-type and $\triangle L A E 1$ strains of $F$. verticillioides demonstrated a reduction in fumonisin pathway expression at 6 days in liquid culture, but no significant differences in fumonisin production at 6 or 11 days. The discrepancy observed between wild-type and $\triangle L A E 1$ fumonisin production described here may be explained by either alternative regulation of toxin production or the different culture conditions (PDA plates in this study; liquid glucose-yeast extract-asparagine-malic acid used by Butchko et al. 2012). These data suggest that antagonism-induced fumonisin production relies on an LAE1-mediated pathway and highlight multifactorial control of fumonisin production.

As specific lipopeptide class production has been shown to respond to the presence of target organisms, it is likely that selection pressures the antibiotic repertoire of a given strain to be effective against commonly encountered microbes (Leaes et al. 2016). Since $F$. verticillioides and B. mojavensis RRC101 have been observed to colonize maize endophytically, details of their antagonism and underlying mechanisms may produce insight into relationships and interactions of plant microbiota. More detailed studies of the biochemical and transcriptional response of phytopathogens to biocontrol agents are necessary to better understand the mechanisms underlying complex microbial interactions taking place in the phyllospheres of maize and other economically important crops. As lipopeptideproducing Bacillus strains have been observed to aid nematodes in defense against pathogens (Iatsenko et al. 2014), such findings as presented here may hold significance for microbiotic interactions beyond the realm of the phyllosphere.

\section{MATERIALS AND METHODS}

Microbe maintenance and culture conditions.

Bacillus mojavensis RRC101 (ATCC 55732) and Fusarium verticillioides M3125 wild type and M3125::GFP (carrying an integrated pCT74 plasmid conferring GFP expression) were kept at $-80^{\circ} \mathrm{C}$ as $15 \%$ glycerol stocks prior to use (Andrie et al. 2005).

B. mojavensis RRC101 was streaked to isolation on LuriaBertani agar plates at $30^{\circ} \mathrm{C}$. Liquid cultures utilized Liu's modified medium at $30^{\circ} \mathrm{C}$, shaken at $220 \mathrm{rpm}$ for $72 \mathrm{~h}$ (Ma et al. 2012). F. verticillioides was cultured at $27^{\circ} \mathrm{C}$ on Acumedia PDA plates (Neogen).

\section{Genome information.}

Details of the sequencing and assembly of the B. mojavensis RRC101 genome are detailed elsewhere; the assembly is available in GenBank under WGS Project ASJT01 (Gold et al. 2014) and through PubSEED under organism number 1329377.3 (Overbeek et al. 2005). Bidirectional BLAST for secondary metabolism clusters was performed using the B. amyloliquefaciens FZB42 genome (CP000560), with supporting evidence provided through the antiSMASH web resource (Weber et al. 2015). Signal peptide identification was conducted through the Phobius web server (Lin et al. 2005).

\section{Preparation of crude extracts.}

Liquid cultures of RRC101 were centrifuged for $30 \mathrm{~min}$ at $9,800 \times g$ and pellets were discarded. Supernatants were brought to $50 \%$ methanol and applied to a Millipore PrepPak C18 column 
$(1 \times 20$ ", packed with 11 " Millipore Waters prep-pak 500/C18, 55 to $105 \mu \mathrm{m})$. Primary fractions were eluted with an A:B system of water/methanol $+1 \%$ formic acid, starting at 50:50 and progressing in $10 \%$ increments $(500-\mathrm{ml}$ volumes) to $100 \%$ B. Fractions were dried in a Buchi E121 Rotavap and were dissolved in methanol at $20 \times$ original culture concentration for bioassays and HPLC analysis.

Antifungal fractions were further separated on a Hewlett Packard 1050 HPLC system, using the same solvent system, progressing from $20 \%$ A ( $10 \%$ methanol $+1 \%$ formic acid) to $100 \% \mathrm{~B}$ (methanol $+1 \%$ formic acid) over $10 \mathrm{~min}$ and maintaining $100 \% \mathrm{~B}$ for $15 \mathrm{~min}$, at a flow rate of $2 \mathrm{ml} / \mathrm{min}$ and injection volume of $50 \mu \mathrm{l}$. Fractionation was conducted using an Agilent ZORBAX Eclipse XDB-C8 column $(4.6 \times 150 \mathrm{~mm}$, $5 \mu \mathrm{m})$, with fractions collected in 30-s intervals using a Gilson fraction collector. Fraction pools were concentrated based on lipopeptide classes and were dried by Rotavap before dissolving in methanol as $100 \times$ culture concentration.

\section{Analysis and identification of lipopeptides.}

Mass spectrometry analysis of RRC101 fractions was performed using the aforementioned column and solvent system, at a flow rate of $200 \mu \mathrm{l} / \mathrm{min}$. HPLC-MS analyses of extracts were conducted using a Finnigan micro AS, Finnigan surveyor MS pump plus, and Finnigan LCQ duo (Thermo Scientific). This analysis was conducted in positive ion mode, utilizing an ESI source with $5-\mathrm{kV}$ spray voltage and a capillary temperature of $190^{\circ} \mathrm{C}$. Other instrument conditions were maximized for sensitivity by "tuning" to a direct injection of surfactin A (Sigma-Aldrich). Samples were also analyzed on a Dionex UltiMate 3000 AS attached to a Dionex UltiMate 3000 UHPLC $^{+}$ pump, in tandem with a Thermo Scientific LTQ XL. Conditions were the same as above, except the instrument was "tuned" by direct injection of iturin A (Sigma-Aldrich). Commercially obtained Surfactin A (Sigma-Aldrich) was employed as a standard for retention and fragmentation patterns of surfactins, with previously published data used to guide fengycin characterization (Bie et al. 2009; Pathak et al. 2012).

\section{Antagonism assays.}

Fractions were screened for antifungal activity through a plate growth assay. Lipopeptide extracts $(10 \times$ and $20 \times$ as well as a methanol blank) were applied as $50-\mu 1$ spots to the surface of PDA plates, with plates kept open in a sterile laminar-flow hood to fully evaporate the solvent. Five-millimeter plugs of 5 -day-old $F$. verticillioides plate cultures were placed central to extracts at a distance of $2 \mathrm{~cm}$ from any spot. Plates were wrapped with parafilm, were inverted, and were incubated in the dark for 3 days at $27^{\circ} \mathrm{C}$ before evaluation. For microscopic evaluation, assays were conducted as described, with sterile dialysis membrane (14 kDa molecular weight cut-off) overlaid prior to inoculation; at the end of the assay, membranes were lifted from the plates and were cut and transferred to glass slides for staining and visualization.

Mycotoxin assays utilized wild-type strain M3125, with 5-mm plugs collected from the leading edges of colonies as well as the immediately adjacent uncolonized agar to account for potential differences in export efficiency as part of antagonism. Fumonisin extractions from 3-day-old agar plugs were conducted using in $1: 1$ acetonitrile/water with $5 \%$ formic acid, shaken for $2 \mathrm{~h}$ at room temperature, as per Glenn et al. (2008). Extracts were then diluted to $25 \%$ acetonitrile before quantitative analysis via LC-MS, using $\mathrm{FB}_{1}, \mathrm{FB}_{2}$, and $\mathrm{FB}_{3}$ standards for comparison. Statistical analyses were conducted using the $\mathrm{R} \times 64$ statistical software environment (Ihaka and Gentleman 1996).

Digital photographs of assay plates were captured in a Protein Simple FluorChem HD2 system, with antagonism assayed digitally. Briefly, photo files were analyzed in silico (using the GNU image manipulation program) and the distance from colony center to leading edge proximal to each spot was measured. Antagonism was calculated as the percent difference in radial growth between a negative control (methanol) and the original fraction, with growth reduction from fractions averaged and treated as a percentage of reduction compared with the original antagonistic fraction $\left(100 \times\right.$ [negative control $-2^{\circ}$ fraction] $/[$ negative control original antagonistic fraction]).

\section{Staining and microscopy.}

Dialysis membrane sections from antagonism plate assays were transferred to microscope slides, were flooded with stain solutions, and were incubated in the dark for $60 \mathrm{~min}$ at room temperature. Distilled water was used to rinse excess stain before visualization. Calcofluor white was used at a concentration of $1 \mu \mathrm{g} / \mathrm{ml}$ to visualize fungal cell walls, CMAC was used for vacuole imaging at $100 \mu \mathrm{M}$, and FM4-64 was used at $17 \mu \mathrm{M}$ as a vital stain based on visualizing membrane permeability.

Cultures were visualized using a Zeiss Axio Imager.A2 microscope, with an attached AxioCam ERc 5s for digital capture, using Zeiss Zen software for image processing. Fluorescence was observed using a Lumen Dynamics X-Cite 120Q light source, with Zeiss filter sets 20 (Rhodamine, for FM4-64), 38 (cGFP) and 49 (DAPI, for Calcofluor and CMAC).

\section{LITERATURE CITED}

Ariño, A., Gloria, E., Teresa, J., and Antonio, H. 2007. Estimation of dietary intakes of fumonisins B1 and B2 from conventional and organic corn. Food Contr. 18:1058-1062.

Andrie, R. M., Martinez, J. P., and Ciuffetti, L. M. 2005. Development of ToxA and ToxB promoter-driven fluorescent protein expression vectors for use in filamentous ascomycetes. Mycologia 97:1152-1161.

Bacon, C. W., and Hinton, D. M. 2002. Endophytic and biological control potential of Bacillus mojavensis and related species. Biol. Control 23: 274-284.

Bacon, C. W., and Hinton, D. M. 2011a. In planta reduction of maize seedling stalk lesions by the bacterial endophyte Bacillus mojavensis. Can. J. Microbiol. 57:485-492.

Bacon, C. W., and Hinton, D. M. 2011b. Bacillus mojavensis: Its endophytic nature, the surfactins and their role in the plant response to infection by Fusarium verticillioides. Pages 21-39 in: Bacteria in Agrobiology: Plant Growth Responses. D. K. Maheshwari, ed. Springer-Verlag, Berlin.

Bais, H. P., Fall, R., and Vivanco, J. M. 2004. Biocontrol of Bacillus subtilis against infection of Arabidopsis roots by Pseudomonas syringae is facilitated by biofilm formation and surfactin production. Plant Physiol 134:307-319

Batte, M. T., Hooker, N. H., Haab, T. C., and Beaverson, J. 2007. Putting their money where their mouths are: Consumer willingness to pay for multi-ingredient, processed organic food products. Food Policy 32: 145-159.

Bie, X., Lu, Z., and Lu, F. 2009. Identification of fengycin homologues from Bacillus subtilis with ESI-MS/CID. J. Microbiol. Methods 79 272-278.

Bok, J. W., and Keller, N. P. 2004. LaeA, a regulator of secondary metabolism in Aspergillus spp. Eukaryot. Cell 3:527-535.

Bonmatin, J.-M., Laprévote, O., and Peypoux, F. 2003. Diversity among microbial cyclic lipopeptides: Iturins and surfactins. Activity-structure relationships to design new bioactive agents. Comb. Chem. High Throughput Screen. 6:541-556.

Bothast, R. J., Bennett, G. A., Vancauwenberge, J. E., and Richard, J. L. 1992. Fate of Fumonisin B(1) in Naturally Contaminated Corn during Ethanol Fermentation. Appl. Environ. Microbiol. 58:233-236.

Butchko, R. A., Brown, D. W., Busman, M., Tudzynski, B., and Wiemann, P. 2012. Lae1 regulates expression of multiple secondary metabolite gene clusters in Fusarium verticillioides. Fungal Genet. Biol. 49: 602-612.

Butchko, R. A. E., McCormick, S. P., Busman, M., Tudyznski, B., and Wiemann, P. 2011. Regulation of secondary metabolite production in Fusarium species by the global regulator LAE1. Meeting abstract. USDA Agricultural Research Service, Ames, IA, U.S.A. 
Butchko, R. A. E., Plattner, R. D., and Proctor, R. H. 2006. Deletion analysis of FUM genes involved in tricarballylic ester formation during fumonisin biosynthesis. J. Agric. Food Chem. 54:9398-9404.

Cawoy, H., Bettiol, W., Fickers, P., and Ongena, M. 2011 Bacillus-based biological control of plant diseases. Pages 273-302 in: Pesticides in the Modern World-Pesticides Use and Management. M. Stoytcheva, ed. InTech, Rijeka, Croatia.

Chanda, A., Roze, L. V., Kang, S., Artymovich, K. A., Hicks, G. R. Raikhel, N. V., Calvo, A. M., and Linz, J. E. 2009. A key role for vesicles in fungal secondary metabolism. Proc. Natl. Acad. Sci. U.S.A. 106: 19533-19538.

Choi, Y. E., and Xu, J. R. 2010. The cAMP signaling pathway in Fusarium verticillioides is important for conidiation, plant infection, and stress responses but not fumonisin production. Mol. Plant-Microbe Interact 23: 522-533.

Collmer, A., and Keen, N. T. 1986. The role of pectic enzymes in plant pathogenesis. Annu. Rev. Phytopathol. 24:383-409.

Daren Mueller, K. W. 2014. Corn Disease Loss Estimates From the United States and Ontario, Canada-2012. In: Diseases of Corn. Purdue Extension publication BP-96-12-W, Purdue University, West Lafayette, IN, U.S.A.

Davies, J., Spiegelman, G. B., and Yim, G. 2006. The world of subinhibitory antibiotic concentrations. Curr. Opin. Microbiol. 9:445-453.

Deleu, M., Paquot, M., and Nylander, T. 2008. Effect of fengycin, a lipopeptide produced by Bacillus subtilis, on model biomembranes. Biophys. J. 94:2667-2679.

Dertz, E. A., Xu, J., Stintzi, A., and Raymond, K. N. 2006. Bacillibactinmediated iron transport in Bacillus subtilis. J. Am. Chem. Soc. 128:2.

Deshmukh, R., Mathew, A., and Purohit, H. J. 2014. Characterization of antibacterial activity of bikaverin from Fusarium sp. HKF15. J. Biosci. Bioeng. 117:443-448.

Druzhinina, I. S., Seidl-Seiboth, V., Herrera-Estrella, A., Horwitz, B. A. Kenerley, C. M., Monte, E., Mukherjee, P. K., Zeilinger, S., Grigoriev, I. V., and Kubicek, C. P. 2011. Trichoderma: The genomics of opportunistic success. Nat. Rev. Microbiol. 9:749-759.

Geetha, I., and Manonmani, A. M. 2010. Surfactin: A novel mosquitocidal biosurfactant produced by Bacillus subtilis ssp. subtilis (VCRC B471) and influence of abiotic factors on its pupicidal efficacy. Lett. Appl. Microbiol. 51:406-412.

Georgelis, N., Nikolaidis, N., and Cosgrove, D. J. 2014. Biochemical analysis of expansin-like proteins from microbes. Carbohydr. Polym. 100:17-23.

Glenn, A. E., Zitomer, N. C., Zimeri, A. M., Williams, L. D., Riley, R. T., and Proctor, R. H. 2008. Transformation-mediated complementation of a FUM gene cluster deletion in Fusarium verticillioides restores both fumonisin production and pathogenicity on maize seedlings. Mol. Plant-Microbe Interact. 21:87-97.

Gold, S. E., Blacutt, A. A., Meinersmann, R. J., and Bacon, C. W. 2014 Whole-genome shotgun sequence of Bacillus mojavensis strain RRC101, an endophytic bacterium antagonistic to the mycotoxigenic endophytic fungus Fusarium verticillioides. Genome Announc. 2:e01090-14.

Han, Q., Wu, F., Wang, X., Qi, H., Shi, L., Ren, A., Liu, Q., Zhao, M., and Tang, C. 2015. The bacterial lipopeptide iturins induce Verticillium dahliae cell death by affecting fungal signalling pathways and mediate plant defence responses involved in pathogen-associated molecular pattern-triggered immunity. Environ. Microbiol. 17:1166-1188.

Henry, G., Deleu, M., Jourdan, E., Thonart, P., and Ongena, M. 2011. The bacterial lipopeptide surfactin targets the lipid fraction of the plant plasma membrane to trigger immune-related defence responses. Cell. Microbiol. 13:1824-1837.

Hirooka, K., and Fujita, Y. 2010. Excess production of Bacillus subtilis quercetin 2,3-dioxygenase affects cell viability in the presence of quercetin. Biosci. Biotech. Bioch. 74:1030-1038.

Hu, L. B., Zhang, T., Yang, Z. M., Zhou, W., and Shi, Z. Q. 2009. Inhibition of fengycins on the production of fumonisin B1 from Fusarium verticillioides. Lett. Appl. Microbiol. 48:84-89.

Iatsenko, I., Yim, J. J., Schroeder, F. C., and Sommer, R. J. 2014. B. subtilis GS67 protects $C$. elegans from gram-positive pathogens via fengycinmediated microbial antagonism. Curr. Biol. 24:2720-2727.

Idriss, E. E., Makarewicz, O., Farouk, A., Rosner, K., Greiner, R., Bochow, H., Richter, T., and Borriss, R. 2002. Extracellular phytase activity of Bacillus amyloliquefaciens FZB45 contributes to its plant-growthpromoting effects. Microbiology 148:2097-2109.

Ihaka, R., and Gentleman, R. 1996. R: A language for data analysis and graphics. J. Comput. Graph. Stat. 5:299-314.

Jacobsen, B. B., Zidack, N. K., and Larson, R. January 2010. Bacillus isolates and methods of their use to protect against plant pathogens. United States patent US20120003197 A1.
Jourdan, E., Henry, G., Duby, F., Dommes, J., Barthélemy, J. P., Thonart, P., and Ongena, M. 2009. Insights into the defense-related events occurring in plant cells following perception of surfactin-type lipopeptide from Bacillus subtilis. Mol. Plant-Microbe Interact 22:456-468.

Kerff, F., Amoroso, A., Herman, R., Sauvage, E., Petrella, S., Filée, P., Charlier, P., Joris, B., Tabuchi, A., Nikolaidis, N., and Cosgrove, D. J. 2008. Crystal structure and activity of Bacillus subtilis YoaJ (EXLX1), a bacterial expansin that promotes root colonization. Proc. Natl. Acad. Sci. U.S.A. 105:16876-16881.

Kovtunovych, G., Lar, O., Kamalova, S., Kordyum, V., Kleiner, D., and Kozyrovska, N. 1999. Correlation between pectate lyase activity and ability of diazotrophic Klebsiella oxytoca VN 13 to penetrate into plant tissues. Plant Soil 215:1-6.

Leaes, F. L., Velho, R. V., Caldas, D. G., Ritter, A. C., Tsai, S. M., and Brandelli, A. 2016. Expression of essential genes for biosynthesis of antimicrobial peptides of Bacillus is modulated by inactivated cells of target microorganisms. Res. Microbiol. 167:83-89.

Lee, K., Pan, J. J., and May, G. 2009. Endophytic Fusarium verticillioides reduces disease severity caused by Ustilago maydis on maize. FEMS Microbiol. Lett. 299:31-37.

Leslie, J. F., Summerell, B. A., and Bullock, S., eds. 2006. The Fusarium Laboratory Manual. John Wiley \& Sons, New York.

Li, L., Ma, M., Huang, R., Qu, Q., Li, G., Zhou, J., Zhang, K., Lu, K., Niu, X., and Luo, J. 2012. Induction of chlamydospore formation in fusarium by cyclic lipopeptide antibiotics from Bacillus subtilis C2. J. Chem. Ecol. 38:966-974.

Limón, M. C., Rodríguez-Ortiz, R., and Avalos, J. 2010. Bikaverin production and applications. Appl. Microbiol. Biotechnol. 87:21-29.

Lin, T.-P., Chen, C.-L., Fu, H.-C., Wu, C.-Y., Lin, G.-H., Huang, S.-H., Chang, L.-K., and Liu, S.-T. 2005. Functional analysis of fengycin synthetase FenD. BBA-Gene Struct. Expr. 1730:159-164.

Ma, Z., Wang, N., Hu, J., and Wang, S. 2012. Isolation and characterization of a new iturinic lipopeptide, mojavensin A produced by a marinederived bacterium Bacillus mojavensis B0621A. J. Antibiot. 65:317-322

Maget-Dana, R., Thimon, L., Peypoux, F., and Ptak, M. 1992. Surfactin/ iturin A interactions may explain the synergistic effect of surfactin on the biological properties of iturin A. Biochimie 74:1047-1051.

Marasas, W. F. O., Wehner, F. C., Van Rensberg, S. J., and Van Schalkwyk, D. J. 1981. Mycoflora of corn produced in human esophageal cancer areas in Transkei, South Africa. Phytopathology 71:792-796.

Medentsev, A. G., Arinbasarova, A. Iu., and Akimenko, V. K. 2005. [Biosynthesis of naphthoquinone pigments by fungi of the genus Fusarium]. Prikl. Biokhim. Mikrobiol. 41:573-577.

Melnick, R. L., Zidack, N. K., Bailey, B. A., Maximova, S. N., Guiltinan, M., and Backman, P. A. 2008. Bacterial endophytes: Bacillus spp. from annual crops as potential biological control agents of black pod rot of cacao. Biol. Control 46:46-56.

Menke, J., Weber, J., Broz, K., and Kistler, H. C. 2013. Cellular development associated with induced mycotoxin synthesis in the filamentous fungus Fusarium graminearum. PLoS One 8:e63077.

Miller, J. D., Savard, M. E., Schaafsma, A. W., Seifert, K. A., and Reid, L. M. 1995. Mycotoxin production by Fusarium moniliforme and Fusarium proliferatum from Ontario and occurrence of fumonisin in the 1993 corn crop. Rev. Can. Plant Pathol. 17:233-239.

Munkvold, G. P., McGee, D. C., and Carlton, W. M. 1997. Importance of different pathways for maize kernel infection by Fusarium moniliforme. Phytopathology 87:209-217.

Nair, J. R., Singh, G., and Sekar, V. 2002. Isolation and characterization of a novel Bacillus strain from coffee phyllosphere showing antifungal activity. J. Appl. Microbiol. 93:772-780.

Ongena, M., and Jacques, P. 2008. Bacillus lipopeptides: Versatile weapons for plant disease biocontrol. Trends Microbiol. 16:115-125.

Ongena, M., Jacques, P., Touré, Y., Destain, J., Jabrane, A., and Thonart, P. 2005. Involvement of fengycin-type lipopeptides in the multifaceted biocontrol potential of Bacillus subtilis. Appl. Microbiol. Biotechnol. 69:29-38

Overbeek, R., Begley, T., Butler, R. M., Choudhuri, J. V., Chuang, H. Y., Cohoon, M., de Crécy-Lagard, V., Diaz, N., Disz, T., Edwards, R., Fonstein, M., Frank, E. D., Gerdes, S., Glass, E. M., Goesmann, A. Hanson, A., Iwata-Reuyl, D., Jensen, R., Jamshidi, N., Krause, L., Kubal, M., Larsen, N., Linke, B., McHardy, A. C., Meyer, F., Neuweger, H., Olsen, G., Olson, R., Osterman, A., Portnoy, V., Pusch, G. D., Rodionov, D. A., Rückert, C., Steiner, J., Stevens, R., Thiele, I., Vassieva, O., Ye, Y., Zagnitko, O., and Vonstein, V. 2005. The subsystems approach to genome annotation and its use in the project to annotate 1000 genomes. Nucleic Acids Res. 33:5691-5702.

Pathak, K. V., Keharia, H., Gupta, K., Thakur, S. S., and Balaram, P. 2012. Lipopeptides from the banyan endophyte, Bacillus subtilis K1: Mass 
spectrometric characterization of a library of fengycins. J. Am. Soc. Mass Spectrom. 23:1716-1728.

Pollack, J. K., Harris, S. D., and Marten, M. R. 2009. Autophagy in filamentous fungi. Fungal Genet. Biol. 46:1-8.

Qi, G., Zhu, F., Du, P., Yang, X., Qiu, D., Yu, Z., Chen, J., and Zhao, X. 2010. Lipopeptide induces apoptosis in fungal cells by a mitochondriadependent pathway. Peptides 31:1978-1986.

Ramos, F. A., Takaishi, Y., Shirotori, M., Kawaguchi, Y., Tsuchiya, K., Shibata, H., Higuti, T., Tadokoro, T., and Takeuchi, M. 2006. Antibacterial and antioxidant activities of quercetin oxidation products from yellow onion (Allium cepa) skin. J. Agric. Food Chem. 54:3551-3557.

Reinhold-Hurek, B., and Hurek, T. 2011. Living inside plants: Bacterial endophytes. Curr. Opin. Plant Biol. 14:435-443.

Richards, A., Veses, V., and Gow, N. A. R. 2010. Vacuole dynamics in fungi. Fungal Biol. Rev. 24:93-105.

Roberts, M. S., Nakamura, L. K., and Cohan, F. M. 1994. Bacillus mojavensis sp. nov., distinguishable from Bacillus subtilis by sexual isolation, divergence in DNA sequence, and differences in fatty acid composition. Int. J. Syst. Bacteriol. 44:256-264.

Ruijter, N. C. A. d., Verhees, J., Leeuwen, W. v., and Krol, A. R. d. 2003. Evaluation and comparison of the GUS, LUC and GFP reporter system for gene expression studies in plants. Plant Biol. 5:103-115.

Ryu, C. M., Farag, M. A., Hu, C. H., Reddy, M. S., Kloepper, J. W., and Pare, P. W. 2004. Bacterial volatiles induce systemic resistance in Arabidopsis. Plant Physiol. 134:1017-1026.

Semighini, C. P., Murray, N., and Harris, S. D. 2008. Inhibition of Fusarium graminearum growth and development by farnesol. FEMS Microbiol. Lett. 279:259-264.

Sharon, A., Finkelstein, A., Shlezinger, N., and Hatam, I. 2009. Fungal apoptosis: Function, genes and gene function. FEMS Microbiol. Rev. 33: $833-854$
Shu, H.-Y., Lin, G.-H., Wu, Y.-C., Tschen, J. S.-M., and Liu, S.-T. 2002. Amino acids activated by fengycin synthetase FenE. Biochem. Biophys. Res. Commun. 292:789-793.

Spaepen, S., and Vanderleyden, J. 2011. Auxin and plant-microbe interactions. Cold Spring Harbor Perspect. Biol. 3:a001438.

Studt, L., Wiemann, P., Kleigrewe, K., Humpf, H.-U., and Tudzynski, B. 2012. Biosynthesis of fusarubins accounts for pigmentation of Fusarium fujikuroi perithecia. Appl. Environ. Microbiol. 78:44684480 .

United States Food and Drug Administration. 2001. Guidance for industry: Fumonisin levels in human foods and animal feeds; final guidance. U.S Department of Health and Human Services, Washington, DC

Voss, K. A., Howard, P. C., Riley, R. T., Sharma, R. P., Bucci, T. J., and Lorentzen, R. J. 2002. Carcinogenicity and mechanism of action of fumonisin B1: A mycotoxin produced by Fusarium moniliforme (=F. verticillioides). Cancer Detect. Prev. 26:1-9.

Weber, T., Blin, K., Duddela, S., Krug, D., Kim, H. U., Bruccoleri, R., Lee, S. Y., Fischbach, M. A., Müller, R., Wohlleben, W., Breitling, R., Takano, E., and Medema, M. H. 2015. antiSMASH 3.0-a comprehensive resource for the genome mining of biosynthetic gene clusters. Nucleic Acids Res. 43:W237-W243.

Wegener, C., Bartling, S., Olsen, O., Weber, J., and von Wettstein, D. 1996. Pectate lyase in transgenic potatoes confers pre-activation of defence against Erwinia carotovora. Physiol. Mol. Plant Pathol. 49:359-376.

Xu, D., and Côté, J. C. 2003. Phylogenetic relationships between Bacillus species and related genera inferred from comparison of $3^{\prime}$ end $16 \mathrm{~S}$ rDNA and $5^{\prime}$ end 16S-23S ITS nucleotide sequences. Int. J. Syst. Evol. Microbiol. 53:695-704

Zhang, S., Wang, Y., Meng, L., Li, J., Zhao, X., Cao, X., Chen, X., Wang, A., and Li, J. 2012. Isolation and characterization of antifungal lipopeptides produced by endophytic Bacillus amyloliquefaciens TF28. Afr. J. Microbiol. Res. 6:1747-1755. 